University of Nebraska - Lincoln

DigitalCommons@University of Nebraska - Lincoln

Agronomy \& Horticulture - Faculty Publications

Agronomy and Horticulture Department

2012

\title{
Molecular mapping of soybean rust resistance in soybean accession PI 561356 and SNP haplotype analysis of the Rpp1 region in diverse germplasm
}

\author{
Ki-Seung Kim \\ University of Illinois \\ Jair R. Unfried \\ TMG-Tropical Melhoramento \& Genética, Caixa Postal 387, Ltda. Rodovia Celso Garcia Cid, Km 87, Parque \\ Industrial, Cambé, Paraná 86183-600, Brazil \\ D. L. Hyten \\ USDA-ARS, Soybean Genomics and Improvement Laboratory, Beltsville, Maryland, david.hyten@unl.edu \\ Reid D. Frederick \\ Foreign Disease-Weed Science Research Unit, USDA-ARS, reid.frederick@usda.gov \\ Glen L. Hartman \\ University of Illinois \\ Follow this and additional works at: https://digitalcommons.unl.edu/agronomyfacpub

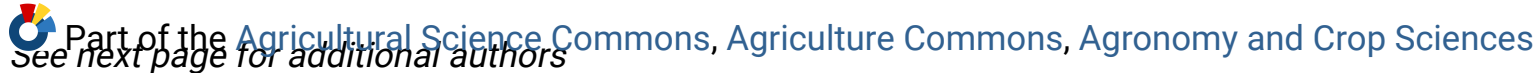 \\ Commons, Botany Commons, Horticulture Commons, Other Plant Sciences Commons, and the Plant \\ Biology Commons
}

Kim, Ki-Seung; Unfried, Jair R.; Hyten, D. L.; Frederick, Reid D.; Hartman, Glen L.; Nelson, Randall L.; Song, Qijian; and Diers, Brian W., "Molecular mapping of soybean rust resistance in soybean accession PI 561356 and SNP haplotype analysis of the Rpp1 region in diverse germplasm" (2012). Agronomy \& Horticulture -- Faculty Publications. 805.

https://digitalcommons.unl.edu/agronomyfacpub/805

This Article is brought to you for free and open access by the Agronomy and Horticulture Department at DigitalCommons@University of Nebraska - Lincoln. It has been accepted for inclusion in Agronomy \& Horticulture -Faculty Publications by an authorized administrator of DigitalCommons@University of Nebraska - Lincoln. 
Authors

Ki-Seung Kim, Jair R. Unfried, D. L. Hyten, Reid D. Frederick, Glen L. Hartman, Randall L. Nelson, Qijian Song, and Brian W. Diers 


\title{
Molecular mapping of soybean rust resistance in soybean accession PI 561356 and SNP haplotype analysis of the Rpp1 region in diverse germplasm
}

\author{
Ki-Seung Kim · Jair R. Unfried • David L. Hyten • \\ Reid D. Frederick · Glen L. Hartman • \\ Randall L. Nelson · Qijian Song · Brian W. Diers
}

Received: 23 February 2012 / Accepted: 28 June 2012 / Published online: 27 July 2012

(C) Springer-Verlag 2012

\begin{abstract}
Soybean rust (SBR), caused by Phakopsora pachyrhizi Sydow, is one of the most economically important and destructive diseases of soybean [Glycine $\max$ (L.) Merr.] and the discovery of novel SBR resistance genes is needed because of virulence diversity in the pathogen. The objectives of this research were to map SBR resistance in plant introduction (PI) 561356 and to identify single nucle-
\end{abstract}

Communicated by H. T. Nguyen.

K.-S. Kim · B. W. Diers ( $ه)$

Department of Crop Science, University of Illinois,

1101 W. Peabody Drive, Urbana, IL 61801, USA

e-mail: bdiers@illinois.edu

J. R. Unfried

TMG-Tropical Melhoramento \& Genética, Caixa Postal 387, Ltda. Rodovia Celso Garcia Cid, Km 87, Parque Industrial,

Cambé, Paraná 86183-600, Brazil

D. L. Hyten

Soybean Genomics and Improvement Laboratory,

USDA-ARS, Beltsville, MD 20705, USA

Present Address:

D. L. Hyten

Pioneer Hi-Bred, Johnston, IA 50131, USA

R. D. Frederick

Foreign Disease-Weed Science Research Unit, USDA-ARS,

1301 Ditto Avenue, Fort Detrick, MD 21702, USA

G. L. Hartman · R. L. Nelson

Soybean/Maize Germplasm, Pathology, and Genetics Research

Unit, Department of Crop Sciences, University of Illinois,

USDA-ARS, Urbana, IL 61801, USA

Q. Song

Soybean Genomics and Improvement Laboratory, Beltsville Agricultural Research Center, USDA-ARS,

Beltsville, MD 20705, USA otide polymorphism (SNP) haplotypes within the region on soybean chromosome 18 where the SBR resistance gene Rpp1 maps. One-hundred $\mathrm{F}_{2: 3}$ lines derived from a cross between PI 561356 and the susceptible experimental line LD02-4485 were genotyped with genetic markers and phenotyped for resistance to $P$. pachyrhizi isolate ZM01-1. The segregation ratio of reddish brown versus tan lesion type in the population supported that resistance was controlled by a single dominant gene. The gene was mapped to a $1-\mathrm{cM}$ region on soybean chromosome 18 corresponding to the same interval as Rppl. A haplotype analysis of diverse germplasm across a 213-kb interval that included Rppl revealed 21 distinct haplotypes of which 4 were present among 5 SBR resistance sources that have a resistance gene in the Rppl region. Four major North American soybean ancestors belong to the same SNP haplotype as PI 561356 and seven belong to the same haplotype as PI 594538A, the $R p p 1-b$ source. There were no North American soybean ancestors belonging to the SNP haplotypes found in PI 200492, the source of Rppl, or PI 587886 and PI 587880A, additional sources with SBR resistance mapping to the Rppl region.

\section{Introduction}

Soybean rust (SBR) is caused by the fungus Phakopsora pachyrhizi Sydow and is one of the most economically important soybean diseases worldwide. SBR was first identified in Japan in 1902 (Hennings 1903), Hawaii in 1994 (Killgore and Heu 1994), and Brazil in 2001 (Yorinori et al. 2005). After SBR was first discovered in the continental USA in plots at the Louisiana State University research station in 2004 (Schneider et al. 2005), the disease spread to 20 US states, to Ontario in Canada, and to 9 states in 
Mexico (Isard et al. 2005; Hershman et al. 2011). Most soybean cultivars grown in the USA are highly susceptible to SBR, which could lead to epidemics if weather conditions are conducive to disease development (Miles et al. 2003).

$P$. pachyrhizi infects more than 150 species of plants from more than 53 genera including soybean, related Glycine species, and other hosts in the Fabaceae (Hartman et al. 2011). This broad host range is unusual among rust pathogens and may be the result of genes that contribute to a diverse and complex virulence pattern (Hartman et al. 2005). The ideal conditions triggering infection are $10 \mathrm{~h}$ of moisture (rain, dew, or irrigation) on the leaf surface and day temperatures ranging from 15 to $28^{\circ} \mathrm{C}$ (optimal $22-23^{\circ} \mathrm{C}$ ) (Ribeiro et al. 2007). Disease development is suppressed when temperatures exceed $30{ }^{\circ} \mathrm{C}$ (Bromfield 1984). As the disease progresses, the leaf tissue around the infected regions become pale brown (TAN reaction) in susceptible genotypes or reddish brown (RB reaction) in incompletely resistant genotypes (Miles et al. 2011). In the case of Rppl from plant introduction (PI) 200492, no lesions develop, and this resistance is referred to as an immune (IM) response in the presence of certain isolates (Miles et al. 2011).

Soybean yield losses up to $80 \%$ in experimental trials have been reported in Asia (Hartman et al. 1991) and $63 \%$ have been reported in Brazil during 2003, $60 \%$ in Paraguay during 2001 (Yorinori et al. 2005), up to $100 \%$ in South Africa (Caldwell and McLaren 2004), and up to $55 \%$ in the USA (Mueller et al. 2009). Because commercial soybean cultivars resistant to SBR are not available in the USA, fungicide applications are the only method currently available to control the disease. Fungicide applications result in significant production cost increases and environmental contamination. The cost of an individual fungicide application is estimated to be from $\$ 37$ to $\$ 50$ per ha and two or three applications may be needed over the course of a growing season (Born and Diver 2005).

The development and production of SBR-resistant cultivars could reduce losses caused by the disease without the expense and negative environmental impact of fungicide applications. Over the last decade, there has been a significant effort to find sources of resistance to SBR. More than 16,000 accessions from the USDA Soybean Germplasm Collection have been screened for SBR resistance with a mixture of $P$. pachyrhizi isolates in greenhouse tests (Miles et al. 2006). No US commercial cultivars evaluated were found to have SBR resistance in these tests; however, 805 accessions were identified with resistance and needing further characterization.

SBR resistance alleles at six loci have been identified and mapped. Rppl from PI 200492 (Hyten et al. 2007), $R p p 1-b$ from PI 594538A (Chakraborty et al. 2009) and SBR resistance genes from PI 587886 and PI 587880A
(Ray et al. 2009) were mapped to the same region on soybean chromosome 18 [linkage group (LG) G]. Rpp2 (Silva et al. 2008) was mapped on chromosome 16 (LG J), Rpp3 (Hyten et al. 2009) and Rpp?(Hyuuga) (Monteros et al. 2007) were mapped on chromosome 6 (LG C2), Rpp4 (Silva et al. 2008) and Rpp6 (Li et al. 2012) were mapped to different regions than Rppl on chromosome 18 (LG G), and Rpp 5 (Garcia et al. 2008) was mapped on chromosome 3 (LG N). Due to the high virulence variability of $P$. pachyrhizi isolates, Rppl, Rppl-b, and Rpp3 already have been defeated in the field in Brazil (Ribeiro et al. 2007; Yorinori et al. 2005). This shows that SBR resistance genes are not durable and it is important to discover additional resistance genes in soybean.

Marker-assisted selection (MAS) can result in increased genetic gains in breeding programs through the indirect selection of gene or genes with genetic markers (Pathan and Sleper 2008). Linkage disequilibrium (LD) between genetic markers and target genes provides a basic principle of MAS that marker alleles are not randomly associated with target gene alleles (Utomo and Linscombe 2009). Single nucleotide polymorphisms (SNPs) are a useful tool to quantify LD, and the analysis of SNP haplotypes has been the focus of recent studies (Zhu et al. 2003). There are several advantages of SNP markers over other genetic marker types. These advantages include: SNPs are the most abundant form of genetic variation within genomes and a wide array of technologies have been developed for high throughput SNP analysis (Zhu et al. 2003; Fan et al. 2006). A SNP haplotype refers to a distinct combination of SNPs that are tightly linked in a region of a chromosome (Shastry 2004) or a distinct combination of SNPs within LD block which tend to be inherited as an entire unit from a parent to its progeny. Information provided by SNPs is most useful when several closely spaced SNPs completely define haplotypes in the region being examined (Johnson et al. 2001). SNPs that can differentiate haplotypes have been called 'haplotype tags' (Johnson et al. 2001) and can be used as important genetic markers for MAS and genetic mapping.

The soybean genome has a relatively high LD compared to other plant species. The estimated average distance at which LD decays to half of its maximum value in cultivated soybean is approximately $150 \mathrm{~kb}$ and in wild soybean (Glycine soja Sieb. and Zucc) $75 \mathrm{~kb}$ (Lam et al. 2010). In contrast, similar levels of LD decay were estimated to occur at $<1 \mathrm{~kb}$ in maize (Zea mays L.) and wild and cultivated rice (Oryza sativa L.) (Gore et al. 2009; Zhu et al. 2007). The percentage and total long $\mathrm{LD}(>150 \mathrm{~kb})$ in cultivated soybean $(1.5 \%$, total length $57.7 \mathrm{Mb})$ were higher than in wild soybeans $(0.6 \%$, total length $35.7 \mathrm{Mb})$ and the longest $\mathrm{LD}$ block in cultivated soybean was $\sim 1 \mathrm{Mb}$, whereas the longest LD block in wild soybeans was $\sim 500 \mathrm{~kb}$ (Lam et al. 2010). The high LD in soybean is likely the result of 
domestication bottlenecks, the inbreeding nature of the crop, and selective breeding (Hyten et al. 2006). In the presence of high LD, a small subset of SNP haplotype tags may be sufficient to define the haplotypes completely (Rafalski 2002).

The first objective of this study was to determine the mode of inheritance and map the location of SBR resistance gene or genes in soybean PI 561356. The second objective was to identify SNP haplotypes within the Rppl region where resistance from PI 561356 maps. This genetic mapping and SNP haplotype analysis will be useful for determining genetic variation in the Rppl region on soybean chromosome 18, for identifying SSR and SNP markers closely linked to the resistance genes, and for studying the association between SNP haplotypes and SBR resistance in the Rppl region in soybean.

\section{Materials and methods}

\section{Plant material}

A population of $100 \mathrm{~F}_{2: 3}$ lines derived from a cross between PI 561356 and LD02-4485 was used for genetic mapping of SBR resistance. PI 561356 is a maturity group (MG) V soybean accession originating from Zhejiang, China (USDA-ARS 2012). PI 561356 showed a mixed lesion type (RB and TAN) to a mixture of $P$. pachyrhizi isolates from Thailand (TH01-1), Brazil (BZ01-1), Paraguay (PG01-2), and Zimbabwe (ZM01-1) (Miles et al. 2006). LD02-4485 is a high-yielding MG II experimental line developed by the University of Illinois that is susceptible to SBR, but resistant to soybean cyst nematode (Heterodera glycines Ichinohe).

Four soybean accessions, PI 200492 (Rppl), PI 594538A (Rppl-b), PI 587886 and PI 587880A, with SBR resistance genes that mapped to the Rppl region on chromosome 18 (Hyten et al. 2007; Chakraborty et al. 2009; Ray et al. 2009), PI 561356, the cultivar Williams 82 as well as 33 major North American soybean ancestors that contribute at least $95 \%$ of the alleles in North America cultivars released from 1947 and 1988 (Gizlice et al. 1994) were used for SNP haplotype analysis in a 213-kb interval surrounding Rppl (Table 1). Seeds of the accessions were obtained from the USDA Soybean Germplasm Collection (Urbana, IL, USA).

\section{P. pachyrhizi isolate evaluation}

The $100 \mathrm{~F}_{2: 3}$ lines were tested for SBR resistance at the USDA-ARS Foreign Disease-Weed Science Research Unit (FDWSRU), Plant Pathogen Containment Facility at Fort Detrick, MD (Melching et al. 1983), under the appropriate permit from the USDA Animal Plant Health Inspection Service. The population was arranged in a randomized complete block design with ten replicates. The experiments included the following known resistant and susceptible checks: PI 200492 (Rpp1), L85-2378 (Rpp1), PI 230970 (Rpp2), PI 462312 (Rpp3), PI 459025 (Rpp4), G01-PR33 [which carries the SBR resistance gene Rpp?(Hyuuga)], and the cultivar Williams (susceptible). The test was initiated by sowing two seeds per cell in flats $(6 \times 12$ cells, $27 \times 52 \mathrm{~cm}$ ) filled with Sunshine LC $_{1}$ mix (Sun Grow Horticultural Products, Belleview, WA).

The lines and checks were inoculated with the $P$. pachyrhizi isolate ZM01-1 collected in Zimbabwe during 2001. This isolate was used as the inoculum source to map resistance from PI 561356, because the PI gave a strong RB response to the isolate. Spores of the isolate were routinely increased on "Williams 82" and stored under liquid nitrogen. Inoculum preparation and plant inoculations were conducted as described by Hyten et al. (2007). After inoculation, plants were incubated for $24 \mathrm{~h}$ at $20^{\circ} \mathrm{C}$ in a dew chamber and then moved to a greenhouse maintained at $20{ }^{\circ} \mathrm{C}$ for 14 days until symptoms were ready to be scored. Two leaflets from the first trifoliate of each inoculated plant were rated for resistant RB type or susceptible TAN type. Disease severity based on symptom and lesion development was rated on a scale of 1 (no visible symptom) to 5 (prolific lesions) as described by Miles et al. (2006). The relative percentage of sporulation was also rated on a single plant basis using a scale of 1 (no sporulation) to 5 (76-100\% of the lesions sporulating) as described by Chakraborty et al. (2009). All TAN lesions were sporulating and given a sporulation rating of 5 .

PI 200492, PI 594538A, PI 587886, PI 587880A, PI 561356, Williams 82, and 11 North American soybean ancestors belonging to SNP haplotype 1 or 9 (Tables 1,2) were evaluated for resistance to the $P$. pachyrhizi isolates FL07-1 collected at Quincy, Florida during 2007 and ZM01-1 to test for an association between SNP haplotypes and SBR resistance (Table 2). The test with FL07-1 was conducted at the USDA-ARS Plant Pathogen Containment Facility at Urbana, IL, and the test with ZM01-1 was conducted at the USDA-ARS FDWSRU Plant Pathogen Containment Facility. For the FL07-1 test, at least 12 plants of each PI and Williams 82 were grown in an 11-cm diameter pot in a non-replicated experiment and inoculations were conducted when the first trifoliolate was fully expanded (V1; Fehr et al. 1971). Urediniospores collected from leaves of Williams 82 were suspended in sterile distilled water containing $0.01 \%$ Tween 20 (sodium monolaurate) and inoculated plants were incubated inside a dew chamber set at $20^{\circ} \mathrm{C}$ for $24 \mathrm{~h}$ (Pham et al. 2009). For the ZM01-1 test, two replications of 12 plants of each PI and Williams 82 were tested and inoculations were conducted as 
Table 1 SNP haplotypes and SSR marker alleles for five soybean rust resistance soybean accessions, the cultivar Williams 82 , and 33 major North American soybean ancestors in a 213-kb interval including the Rppl region on soybean chromosome 18

\begin{tabular}{|c|c|c|c|c|c|c|c|c|c|c|c|c|c|c|c|c|c|c|c|c|c|c|c|c|c|c|c|c|}
\hline \multirow[b]{2}{*}{ PI number } & \multirow[b]{2}{*}{$\begin{array}{l}\text { SBR Resistance } \\
\text { gene }\end{array}$} & \multirow[b]{2}{*}{ Origin } & \multirow[b]{2}{*}{ 厄. } & \multirow{2}{*}{ 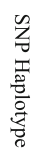 } & \multicolumn{24}{|c|}{ Marker positions ${ }^{\mathrm{b}}(\mathrm{bp})$ on soybean chromosome 18} \\
\hline & & & & & 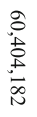 & 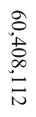 & 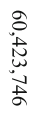 & $\begin{array}{l}8 \\
\text { o } \\
0 \\
-1 \\
-0 \\
0 \\
0 \\
0\end{array}$ & 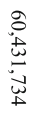 & 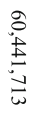 & 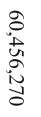 & 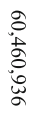 & 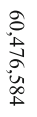 & 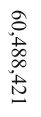 & 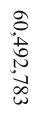 & 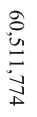 & 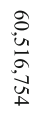 & $\begin{array}{l}\tilde{2} \\
\hat{0} \\
\tilde{O}_{0} \\
0\end{array}$ & $\begin{array}{l}\tilde{D} \\
\hat{\approx} \\
\sigma\end{array}$ & $\begin{array}{l}\stackrel{\partial}{o} \\
\tilde{u} \\
\vec{d} \\
\ddot{\alpha} \\
0\end{array}$ & 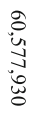 & 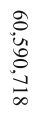 & 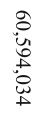 & $\begin{array}{l}\stackrel{2}{ } \\
\text { in } \\
\infty \\
\infty \\
\infty \\
\infty \\
\infty \\
\text { un }\end{array}$ & 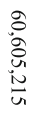 & 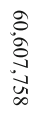 & 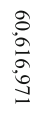 & 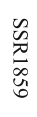 \\
\hline PI 200492 & Rppl & Japan (Shikoku) & & 16 & $\mathrm{C}$ & $\mathrm{T}$ & $\mathrm{C}$ & $G^{\mathrm{d}}$ & $\bar{G}$ & $\overline{\mathrm{A}}$ & $\mathrm{T}$ & $\mathrm{T}$ & $\mathrm{G}$ & $\mathrm{C}$ & $\mathrm{C}$ & $\mathrm{G}$ & $\overline{\mathrm{C}}$ & 1 & 1 & $\mathrm{G}$ & $\mathrm{C}$ & $\mathbf{T}$ & $\mathrm{C}$ & $\mathrm{G}$ & A & $\mathrm{T}$ & $\mathrm{A}$ & 1 \\
\hline PI 594538A & Rpp1-b & China (Fujian) & & 1 & $\mathrm{~T}$ & G & $\mathrm{C}$ & A & $\mathrm{T}$ & G & G & $\mathrm{T}$ & G & $\mathrm{T}$ & $\mathrm{C}$ & A & $\mathrm{C}$ & 2 & 2 & G & $\mathrm{T}$ & $\mathrm{C}$ & $\mathrm{C}$ & $\mathbf{A}$ & A & G & A & 2 \\
\hline PI 587886 & Rpp1-? & China (Zhejiang) & & 19 & $\mathrm{C}$ & $\mathrm{T}$ & $\mathbf{A}$ & A & $\mathrm{G}$ & A & $\mathrm{T}$ & $\mathrm{C}$ & A & $\mathrm{C}$ & $\mathbf{T}$ & G & $\mathbf{N}^{\mathrm{e}}$ & 3 & 2 & G & $\mathrm{C}$ & $\mathrm{C}$ & $\mathrm{C}$ & G & C & $\mathrm{T}$ & A & 2 \\
\hline PI $587880 \mathrm{~A}$ & Rpp1-? & China (Zhejiang) & & 19 & $\mathrm{C}$ & $\mathrm{T}$ & $\mathbf{A}$ & A & $\mathrm{G}$ & A & $\mathrm{T}$ & $\mathrm{C}$ & A & $\mathrm{C}$ & $\mathbf{T}$ & G & $\mathbf{N}$ & 3 & 2 & G & $\mathrm{C}$ & $\mathrm{C}$ & $\mathrm{C}$ & $\mathrm{G}$ & C & $\mathrm{T}$ & A & 2 \\
\hline PI 561356 & Rpp1-? & China (Zhejiang) & & 9 & $\mathrm{~T}$ & G & $\mathrm{C}$ & A & $\mathrm{G}$ & $\mathrm{G}$ & $\mathrm{T}$ & $\mathrm{C}$ & A & $\mathrm{T}$ & $\mathrm{C}$ & G & $\mathrm{C}$ & 2 & 2 & $\mathbf{A}$ & $\mathrm{C}$ & $\mathrm{C}$ & $\mathrm{C}$ & $\mathrm{G}$ & A & G & C & 2 \\
\hline Williams 82 & Susceptible check & China & & 2 & $\mathrm{~T}$ & $\mathrm{G}$ & $\mathrm{C}$ & A & $\mathrm{T}$ & G & G & $\mathrm{T}$ & G & $\mathrm{T}$ & $\mathrm{C}$ & A & $\mathrm{C}$ & 4 & 1 & G & $\mathrm{T}$ & $\mathrm{C}$ & $\mathrm{C}$ & $\mathrm{G}$ & A & G & A & 4 \\
\hline PI number & Cultivar name & & & & & & & & & & & & & & & & & & & & & & & & & & & \\
\hline PI 548406 & Richland & China (Jilin) & 11.3 & 1 & $\mathrm{~T}$ & G & $\mathrm{C}$ & A & $\mathrm{T}$ & G & $\mathrm{G}$ & $\mathrm{T}$ & G & $\mathrm{T}$ & $\mathrm{C}$ & A & $\mathrm{C}$ & 2 & 3 & G & $\mathrm{T}$ & $\mathrm{C}$ & $\mathrm{C}$ & A & A & $\mathrm{G}$ & A & 3 \\
\hline PI 548488 & S-100 & China (Heilongjiang) & 1.8 & 1 & $\mathrm{~T}$ & $\mathrm{G}$ & $\mathrm{C}$ & A & $\mathrm{T}$ & G & G & $\mathrm{T}$ & G & $\mathrm{T}$ & $\mathrm{C}$ & A & $\mathrm{C}$ & 4 & 1 & $\mathrm{G}$ & $\mathrm{T}$ & $\mathrm{C}$ & $\mathrm{C}$ & A & A & G & A & 3 \\
\hline PI 548298 & $\mathrm{AK}$ (Harrow) & na & 6.9 & 1 & $\mathrm{~T}$ & G & $\mathrm{C}$ & A & $\mathrm{T}$ & G & $\mathrm{G}$ & $\mathrm{T}$ & G & $\mathrm{T}$ & $\mathrm{C}$ & A & $\mathrm{C}$ & 4 & 1 & G & $\mathrm{T}$ & $\mathrm{C}$ & $\mathrm{C}$ & A & A & G & A & 3 \\
\hline PI 548318 & Dunfield & China (Jilin) & 3.5 & 1 & $\mathrm{~T}$ & $\mathrm{G}$ & $\mathrm{C}$ & A & $\mathrm{T}$ & $\mathrm{G}$ & $\mathrm{G}$ & $\mathrm{T}$ & G & $\mathrm{T}$ & $\mathrm{C}$ & A & $\mathrm{C}$ & 4 & 3 & $\mathrm{G}$ & $\mathrm{T}$ & $\mathrm{C}$ & $\mathrm{C}$ & A & A & G & A & 3 \\
\hline PI 548484 & Ralsoy & N. Korea (Pyongyang) & 0.1 & 1 & $\mathrm{~T}$ & G & $\mathrm{C}$ & A & $\mathrm{T}$ & $\mathrm{G}$ & $\mathrm{G}$ & $\mathrm{T}$ & G & $\mathrm{T}$ & $\mathrm{C}$ & A & $\mathrm{C}$ & 5 & 4 & $\mathrm{G}$ & $\mathrm{T}$ & $\mathrm{C}$ & $\mathrm{C}$ & A & A & G & A & 3 \\
\hline PI 548438 & Arksoy & N. Korea (Pyongyang) & 0.0 & 1 & $\mathrm{~T}$ & G & $\mathrm{C}$ & A & $\mathrm{T}$ & $\mathrm{G}$ & $\mathrm{G}$ & $\mathrm{T}$ & G & $\mathrm{T}$ & $\mathrm{C}$ & A & $\mathrm{C}$ & 5 & 4 & $\mathrm{G}$ & $\mathrm{T}$ & $\mathrm{C}$ & $\mathrm{C}$ & $\mathrm{A}$ & A & $\mathrm{G}$ & $\mathrm{A}$ & 3 \\
\hline PI 240664 & Bilomi No.3 & Philippines (Luzon) & 0.0 & 1 & $\mathrm{~T}$ & G & $\mathrm{C}$ & A & $\mathrm{T}$ & $\mathrm{G}$ & $\mathrm{G}$ & $\mathrm{T}$ & G & $\mathrm{T}$ & $\mathrm{C}$ & A & $\mathrm{C}$ & 4 & 4 & $\mathrm{G}$ & $\mathrm{T}$ & $\mathrm{C}$ & $\mathrm{C}$ & A & A & $\mathrm{G}$ & A & 3 \\
\hline PI 548360 & Korean & N. Korea & 0.8 & 2 & $\mathrm{~T}$ & G & $\mathrm{C}$ & A & $\mathrm{T}$ & $\mathrm{G}$ & $\mathrm{G}$ & $\mathrm{T}$ & G & $\mathrm{T}$ & $\mathrm{C}$ & A & $\mathrm{C}$ & 1 & 5 & $\mathrm{G}$ & $\mathrm{T}$ & $\mathrm{C}$ & $\mathrm{C}$ & $\mathrm{G}$ & A & $\mathrm{G}$ & A & 3 \\
\hline PI 548311 & Capital & China (Heilongjiang) & 2.4 & 2 & $\mathrm{~T}$ & G & $\mathrm{C}$ & A & $\mathrm{T}$ & $\mathrm{G}$ & $\mathrm{G}$ & $\mathrm{T}$ & G & $\mathrm{T}$ & $\mathrm{C}$ & A & $\mathrm{C}$ & 2 & 5 & $\mathrm{G}$ & $\mathrm{T}$ & $\mathrm{C}$ & $\mathrm{C}$ & $\mathrm{G}$ & A & $\mathrm{G}$ & A & 3 \\
\hline PI 548348 & Illini & China & 3.1 & 3 & $\mathrm{~T}$ & G & $\mathrm{C}$ & A & $\mathrm{T}$ & G & $\mathrm{G}$ & $\mathrm{T}$ & G & $\mathrm{T}$ & $\mathrm{C}$ & A & $\mathrm{C}$ & 1 & 1 & $\mathrm{G}$ & $\mathrm{T}$ & $\mathrm{C}$ & $\mathrm{C}$ & $\mathrm{A}$ & A & $\mathrm{N}$ & A & 3 \\
\hline PI 548362 & Lincoln & China $(1$ & 24.2 & 4 & $\mathrm{~T}$ & G & $\mathrm{C}$ & A & $\mathrm{T}$ & $\mathrm{G}$ & G & $\mathrm{T}$ & G & $\mathrm{T}$ & $\mathrm{N}$ & A & $\mathrm{C}$ & 1 & 1 & G & $\mathrm{T}$ & $\mathrm{C}$ & $\mathrm{C}$ & A & A & $\mathrm{N}$ & A & 3 \\
\hline PI 548379 & Mandarin (Ottawa) & China (Heilo & 17.2 & 5 & $\mathrm{~T}$ & G & $\mathrm{C}$ & A & $\mathrm{T}$ & G & G & $\mathrm{T}$ & G & $\mathrm{T}$ & $\mathrm{N}$ & A & $\mathrm{C}$ & 2 & 5 & G & $\mathrm{T}$ & $\mathrm{C}$ & $\mathrm{C}$ & $\mathrm{G}$ & A & $\mathrm{N}$ & A & 3 \\
\hline PI 438471 & Fiskeby III & Sweden (Ostergotland) & 0.7 & 6 & $\mathrm{~T}$ & G & $\mathrm{C}$ & A & $\mathrm{T}$ & $\mathrm{G}$ & G & $\mathrm{T}$ & G & $\mathrm{T}$ & $\mathrm{C}$ & $\mathrm{N}$ & $\mathrm{C}$ & 5 & 6 & A & $\mathrm{C}$ & $\mathrm{C}$ & $\mathrm{C}$ & $\mathrm{G}$ & $\mathrm{C}$ & G & A & 4 \\
\hline PI 548456 & Haberlandt & N. Korea (Pyongyang) & 0.1 & 6 & $\mathrm{~T}$ & G & $\mathrm{C}$ & A & $\mathrm{T}$ & $\mathrm{G}$ & $\mathrm{G}$ & $\mathrm{T}$ & G & $\mathrm{T}$ & $\mathrm{C}$ & G & $\mathrm{N}$ & 3 & 3 & A & $\mathrm{C}$ & $\mathrm{C}$ & $\mathrm{C}$ & G & $\mathrm{C}$ & $\mathrm{G}$ & A & 5 \\
\hline PI 438477 & $840-7-3$ & tland) & 1.1 & 7 & $\mathrm{~T}$ & G & $\mathrm{C}$ & A & $\mathrm{T}$ & $\mathrm{G}$ & G & $\mathrm{T}$ & A & $\mathrm{T}$ & $\mathrm{T}$ & $\mathrm{N}$ & $\mathrm{C}$ & 5 & 6 & A & $\mathrm{C}$ & $\mathrm{C}$ & $\mathrm{C}$ & G & $\mathrm{N}$ & $\mathrm{T}$ & A & 4 \\
\hline PI 088788 & PI 88788 & China (Liaor & 0.4 & 8 & $\mathrm{~T}$ & G & $\mathrm{C}$ & A & $\mathrm{T}$ & G & $\mathrm{T}$ & $\mathrm{T}$ & A & $\mathrm{T}$ & $\mathrm{C}$ & $\mathrm{N}$ & $\mathrm{C}$ & 6 & 7 & G & $\mathrm{T}$ & $\mathrm{C}$ & $\mathrm{C}$ & G & $\mathrm{C}$ & G & $\mathrm{C}$ & 3 \\
\hline PI 548485 & Roanoke & China (Jiangsu & 0.2 & 8 & $\mathrm{~T}$ & G & $\mathrm{C}$ & A & $\mathrm{T}$ & $\mathrm{G}$ & $\mathrm{T}$ & $\mathrm{T}$ & A & $\mathrm{T}$ & $\mathrm{C}$ & $\mathrm{N}$ & $\mathrm{C}$ & 6 & 7 & G & $\mathrm{T}$ & $\mathrm{C}$ & $\mathrm{C}$ & G & $\mathrm{C}$ & G & $\mathrm{C}$ & 3 \\
\hline PI 548477 & Ogden & China, Japan & 4.3 & 9 & $\mathrm{~T}$ & G & $\mathrm{C}$ & A & $\mathrm{G}$ & G & $\mathrm{T}$ & $\mathrm{C}$ & A & $\mathrm{T}$ & $\mathrm{C}$ & G & $\mathrm{C}$ & 3 & 3 & A & $\mathrm{C}$ & $\mathrm{C}$ & $\mathrm{C}$ & G & A & G & $\mathrm{C}$ & 3 \\
\hline PI 548302 & Bansei & Japan (Hokkaido) & 1.1 & 9 & $\mathrm{~T}$ & G & $\mathrm{C}$ & A & $\mathrm{G}$ & G & $\mathrm{T}$ & $\mathrm{C}$ & A & $\mathrm{T}$ & $\mathrm{C}$ & G & $\mathrm{C}$ & 5 & 6 & A & $\mathrm{C}$ & $\mathrm{C}$ & $\mathrm{C}$ & G & A & G & $\mathrm{C}$ & 3 \\
\hline PI 548356 & Kanro & N Korea Pyonoy & 1.0 & 9 & $\mathrm{~T}$ & G & $\mathrm{C}$ & A & $\mathrm{G}$ & G & $\mathrm{T}$ & $\mathrm{C}$ & A & $\mathrm{T}$ & $\mathrm{C}$ & G & $\mathrm{C}$ & 5 & 6 & A & $\mathrm{C}$ & $\mathrm{C}$ & $\mathrm{C}$ & G & A & G & $\mathrm{C}$ & 3 \\
\hline PI 548352 & Jogun & N. Korea (Hamgyong) & 0.8 & 9 & $\mathrm{~T}$ & $\mathrm{G}$ & $\mathrm{C}$ & A & $\mathrm{G}$ & $\mathrm{G}$ & $\mathrm{T}$ & $\mathrm{C}$ & A & $\mathrm{T}$ & $\mathrm{C}$ & G & $\mathrm{C}$ & 3 & 3 & A & $\mathrm{C}$ & $\mathrm{C}$ & $\mathrm{C}$ & G & A & $\mathrm{G}$ & $\mathrm{C}$ & 3 \\
\hline FC031745 & FC 31745 & Unknown & 0.0 & 10 & $\mathrm{~T}$ & $\mathrm{~T}$ & $\mathrm{~N}$ & A & $\mathrm{T}$ & A & $\mathrm{T}$ & $\mathrm{T}$ & A & $\mathrm{T}$ & $\mathrm{T}$ & A & A & 4 & 4 & A & $\mathrm{T}$ & $\mathrm{T}$ & A & A & A & $\mathrm{T}$ & A & 6 \\
\hline PI 548445 & $\mathrm{CNS}$ & China (Jiangsu) & 3.0 & 11 & $\mathrm{C}$ & $\mathrm{T}$ & $\mathrm{C}$ & $\mathrm{G}$ & $\mathrm{G}$ & G & $\mathrm{G}$ & $\mathrm{T}$ & A & $\mathrm{C}$ & $\mathrm{N}$ & G & $\mathrm{C}$ & 7 & 3 & G & $\mathrm{T}$ & $\mathrm{N}$ & $\mathrm{C}$ & A & A & $\mathrm{N}$ & A & 3 \\
\hline PI 360955A & Fiskeby V & Sweden (Ostergotla & 0.5 & 12 & $\mathrm{C}$ & $\mathrm{T}$ & $\mathrm{C}$ & G & $\mathrm{G}$ & G & G & $\mathrm{T}$ & A & $\mathrm{C}$ & $\mathrm{C}$ & G & $\mathrm{C}$ & 7 & 7 & G & $\mathrm{C}$ & $\mathrm{C}$ & $\mathrm{C}$ & G & A & G & A & 3 \\
\hline PI 548391 & Mukden & China (Liaoning) & 4.9 & 12 & $\mathrm{C}$ & $\mathrm{T}$ & $\mathrm{C}$ & G & $\mathrm{G}$ & G & $\mathrm{G}$ & $\mathrm{T}$ & A & $\mathrm{C}$ & $\mathrm{C}$ & G & $\mathrm{C}$ & 7 & 7 & G & $\mathrm{C}$ & $\mathrm{C}$ & $\mathrm{C}$ & $\mathrm{G}$ & A & $\mathrm{G}$ & A & 3 \\
\hline PI 180501 & PI 180501 & China & 0.8 & 12 & $\mathrm{C}$ & $\mathrm{T}$ & $\mathrm{C}$ & G & $\mathrm{G}$ & G & G & $\mathrm{T}$ & A & $\mathrm{C}$ & $\mathrm{C}$ & G & $\mathrm{C}$ & 7 & 7 & G & $\mathrm{C}$ & $\mathrm{C}$ & $\mathrm{C}$ & G & A & G & A & 3 \\
\hline PI 548402 & Peking & China (Beijing) & 0.1 & 13 & $\mathrm{C}$ & $\mathrm{T}$ & $\mathrm{C}$ & G & $\mathrm{G}$ & A & $\mathrm{T}$ & $\mathrm{C}$ & A & $\mathrm{C}$ & $\mathrm{C}$ & G & $\mathrm{N}$ & 7 & 8 & G & $\mathrm{C}$ & $\mathrm{C}$ & $\mathrm{C}$ & G & A & $\mathrm{T}$ & A & 6 \\
\hline PI 548461 & Improved Pelican & China (Fujian) & 0.0 & 14 & $\mathrm{C}$ & $\mathrm{T}$ & $\mathrm{C}$ & G & G & A & $\mathrm{T}$ & $\mathrm{C}$ & A & $\mathrm{C}$ & $\mathrm{C}$ & G & A & 1 & 1 & $\mathrm{~N}$ & $\mathrm{C}$ & $\mathrm{C}$ & $\mathrm{C}$ & G & A & G & $\mathrm{C}$ & 3 \\
\hline PI 548382 & Manitoba Brown & Canada & 1.5 & 15 & $\mathrm{C}$ & $\mathrm{T}$ & $\mathrm{C}$ & G & $\mathrm{G}$ & G & $\mathrm{T}$ & $\mathrm{C}$ & A & $\mathrm{C}$ & $\mathrm{C}$ & G & $\mathrm{C}$ & 2 & 7 & G & $\mathrm{C}$ & $\mathrm{C}$ & $\mathrm{C}$ & G & A & G & A & 3 \\
\hline PI 071506 & PI 71506 & bing & 0.1 & 17 & $\mathrm{C}$ & $\mathrm{T}$ & $\mathrm{C}$ & G & $\mathrm{G}$ & A & $\mathrm{T}$ & $\mathrm{C}$ & A & $\mathrm{T}$ & $\mathrm{C}$ & G & A & ${ }^{f}$ & . & $\mathrm{N}$ & $\mathrm{T}$ & $\mathrm{T}$ & $\mathrm{C}$ & $\mathrm{G}$ & $\mathrm{C}$ & $\mathrm{T}$ & A & 6 \\
\hline PI 548657 & Jackson & & 0.2 & 18 & $\mathrm{C}$ & $\mathrm{T}$ & $\mathrm{C}$ & G & $\mathrm{G}$ & A & $\mathrm{T}$ & $\mathrm{C}$ & A & $\mathrm{T}$ & $\mathrm{N}$ & G & A & . & . & $\mathrm{N}$ & $\mathrm{T}$ & $\mathrm{T}$ & $\mathrm{C}$ & G & $\mathrm{C}$ & $\mathrm{T}$ & A & 6 \\
\hline PI 080837 & PI 80837 & Japan (Akita) & 0.0 & 20 & $\mathrm{C}$ & $\mathrm{T}$ & A & A & $\mathrm{G}$ & A & $\mathrm{T}$ & $\mathrm{C}$ & A & $\mathrm{C}$ & $\mathrm{T}$ & G & $\mathrm{N}$ & . & . & $\mathrm{N}$ & $\mathrm{C}$ & $\mathrm{T}$ & A & A & $\mathrm{C}$ & $\mathrm{T}$ & $\mathrm{C}$ & 3 \\
\hline PI 548603 & Perry & China, Japan & 2.1 & 21 & $\mathrm{~N}$ & $\mathrm{~T}$ & $\mathrm{C}$ & G & $\mathrm{G}$ & G & $\mathrm{T}$ & $\mathrm{T}$ & A & $\mathrm{C}$ & $\mathrm{T}$ & G & $\mathrm{C}$ & . & 1 & $\mathrm{~N}$ & $\mathrm{C}$ & $\mathrm{T}$ & A & A & $\mathrm{C}$ & $\mathrm{T}$ & $\mathrm{C}$ & 3 \\
\hline
\end{tabular}

${ }^{a}$ Percentage of contribution of each PI to the northern soybean varieties from Gizlice et al. (1994)

${ }^{\mathrm{b}}$ Physical position of the markers based on the G. max genome (assembly version 1.01) available at http://soybase.org/gbrowse/cgi-bin/gbrowse/ gmax 1.01/. The base pair (bp) positions of the SNP markers correspond to the locations of each SNP on soybean chromosome 18

c SSR markers used for genetic mapping of the SBR resistance gene in PI 561356. Seven different allele sizes were present for SSR50, eight for SSR66, and six for SSR1859 in the tested 39 soybean accessions

${ }^{\mathrm{d}}$ Boldface indicates SNP markers that can differentiate each haplotype from the other three haplotypes among Rpp1 sources (haplotype tag)

${ }^{\mathrm{e}} \mathrm{N}$ indicates that no genotype could be assigned

${ }^{\mathrm{f}}$ The accession had no PCR product

described by Hyten et al. (2007). The responses to isolates FL07-1 and ZM01-1 (IM, RB or TAN) were evaluated 15 days after inoculation.

\section{Genetic mapping of the SBR resistance in PI 561356}

Genomic DNA from the population was extracted using young trifoliolate leaf tissue pooled from at least ten plants from each line using the CTAB method (hexadecyltrimethylammonium bromide) method described by Keim et al. (1988). To find the position(s) of the resistance gene(s) in PI 561356, bulked segregant analysis (BSA) was used (Michelmore et al. 1991). A resistant bulk was formed by pooling an equal amount of DNA from ten lines with $\mathrm{RB}$ reactions and a susceptible bulk was formed by pooling DNA from ten lines with TAN reactions. The two parents, resistant bulk and susceptible bulk, were first screened with simple sequence repeat (SSR) markers that mapped near Rppl, 2, 3, 4, and 5 . Once the potential locations of resistance genes were identified, the lines in the population were tested with additional markers from these locations. After genetic mapping of the population, DNA from lines (line 9, 64, 71, 96, and 100; Fig. 1) with recombination events near the gene was extracted from at least 20 plants to confirm the initial genotyping results. Primer sequences of the SSR markers were obtained from SoyBase (http:// soybase.org/resources/ssr.php) and Song et al. (2010). 
Table 2 Reactions of 17 soybean accessions to two different Phakopsora pachyrhizi isolates and their SNP haplotypes and alleles for three SSR markers

\begin{tabular}{|c|c|c|c|c|c|c|c|}
\hline \multirow[t]{2}{*}{ PI number } & \multirow[t]{2}{*}{ Resistance gene } & \multirow[t]{2}{*}{ SNP haplotype ${ }^{a}$} & \multirow[t]{2}{*}{ SSR $50^{\mathrm{b}}$} & \multirow[t]{2}{*}{ SSR $66^{\mathrm{b}}$} & \multirow[t]{2}{*}{ SSR $1859^{\mathrm{b}}$} & \multicolumn{2}{|c|}{ P. pachyrhizi isolate } \\
\hline & & & & & & FL07-1 & ZM01-1 \\
\hline PI 200492 & Rppl & 16 & 1 & 1 & 1 & IM & TAN \\
\hline PI 594538A & Rppl-b & 1 & 2 & 2 & 2 & TAN & $\mathrm{RB}$ \\
\hline PI 587886 & Rpp1-? & 19 & 3 & 2 & 2 & TAN & $\mathrm{RB}$ \\
\hline PI 587880A & Rpp1-? & 19 & 3 & 2 & 2 & TAN & $\mathrm{RB}$ \\
\hline PI 561356 & Rpp1-? & 9 & 2 & 2 & 2 & TAN & $\mathrm{RB}$ \\
\hline Williams 82 & Susceptible check & 2 & 4 & 1 & 3 & TAN & TAN \\
\hline PI number & Cultivar name & & & & & & \\
\hline PI 548406 & Richland & 1 & 2 & 3 & 3 & TAN & TAN \\
\hline PI 548488 & S-100 & 1 & 4 & 1 & 3 & TAN & TAN \\
\hline PI 548298 & AK(Harrow) & 1 & 4 & 1 & 3 & TAN & TAN \\
\hline PI 548318 & Dunfield & 1 & 4 & 3 & 3 & TAN & TAN \\
\hline PI 548484 & Ralsoy & 1 & 5 & 4 & 3 & TAN & TAN \\
\hline PI 548438 & Arksoy & 1 & 5 & 4 & 3 & TAN & TAN \\
\hline PI 240664 & Bilomi No.3 & 1 & 4 & 4 & 3 & TAN & TAN \\
\hline PI 548477 & Ogden & 9 & 3 & 3 & 3 & TAN & TAN \\
\hline PI 548302 & Bansei & 9 & 5 & 6 & 3 & TAN & TAN \\
\hline PI 548356 & Kanro & 9 & 5 & 6 & 3 & TAN & TAN \\
\hline PI 548352 & Jogun & 9 & 3 & 3 & 3 & TAN & TAN \\
\hline
\end{tabular}

$I M$ immune response (no visible symptoms; resistant reaction), $R B$ reddish brown-colored lesions (resistant reaction), TAN pale brown-colored lesions (susceptible reaction)

a Haplotypes based on 21 SNP markers within the 213-kb interval including the Rpp1 region on soybean chromosome 18

b SSR markers used for genetic mapping of the SBR resistance gene in PI 561356. Seven different allele sizes were present for SSR50, eight for SSR66, and six for SSR1859 in the tested 39 soybean accessions (Table 1)

Polymerase chain reaction (PCR) and evaluation of PCR products were carried out as previously described by Wang et al. (2003). PCR consisted of 36 cycles of denaturation at $94{ }^{\circ} \mathrm{C}$ for $25-30 \mathrm{~s}$, annealing at $46-62{ }^{\circ} \mathrm{C}$ for 25-30 s, and extension at $68{ }^{\circ} \mathrm{C}$ for $25-30 \mathrm{~s}$ with a PTC 100 Programmable Thermal Controller (MJ Research Inc., Watertown, MA, USA). The PCR products were analyzed by electrophoresis in both $3 \%$ agarose gels (BMA, Rockland, ME, USA) and $6 \%$ nondenaturing polyacrylamide gels (Wang et al. 2003). The polymorphic information content (PIC) values for SSR50, SSR66, and SSR1859 were calculated by using the formula PIC $=1-\sum(P i)^{2}$, where $P i$ represents the proportion of the soybean genotypes carrying the $i^{\text {th }}$ allele (Botstein et al. 1980).

Statistical analysis

The Chi-square tests for SBR lesion type (RB or TAN) and molecular markers were performed to test the goodness of fit of the observed segregation among $\mathrm{F}_{2: 3}$ lines. Linkage analysis was performed to map the location of a gene controlling SBR lesion type (RB or TAN) with JoinMap 3.0
(Van Ooijen and Voorrips 2001). A logarithm (base 10) of the odds (LOD) score of 5.0 was used as a threshold to group markers into a linkage group. Genomic region(s) associated with disease severity was mapped as quantitative trait loci (QTL) using the interval mapping (IM) functions in MapQTL ${ }^{\circledR} 4.0$ (Van Ooijen et al. 2002). The LOD score threshold for declaring a putative locus as significant was determined by 1,000 permutations in MapQTL $^{\circledR}$ 4.0. The gene position was defined as the point of maximum LOD score. Analysis of variance in PROC GLM in SAS 9.2 (SAS Institute 2002) was used to test the significance of the association between lesion type and disease severity. Disease severity and sporulation were also analyzed by PROC GLM in SAS 9.2 (SAS Institute 2002). The means for disease severity and sporulation were separated using the least significant difference (LSD) at $P=0.05$.

SNP haplotype analysis

The SoySNP50 Illumina Infinium chip (Song et al. 2012) was used to genotype the 5 SBR resistance soybean accessions and 33 major North American soybean ancestors in the 213-kb interval including Rppl region 


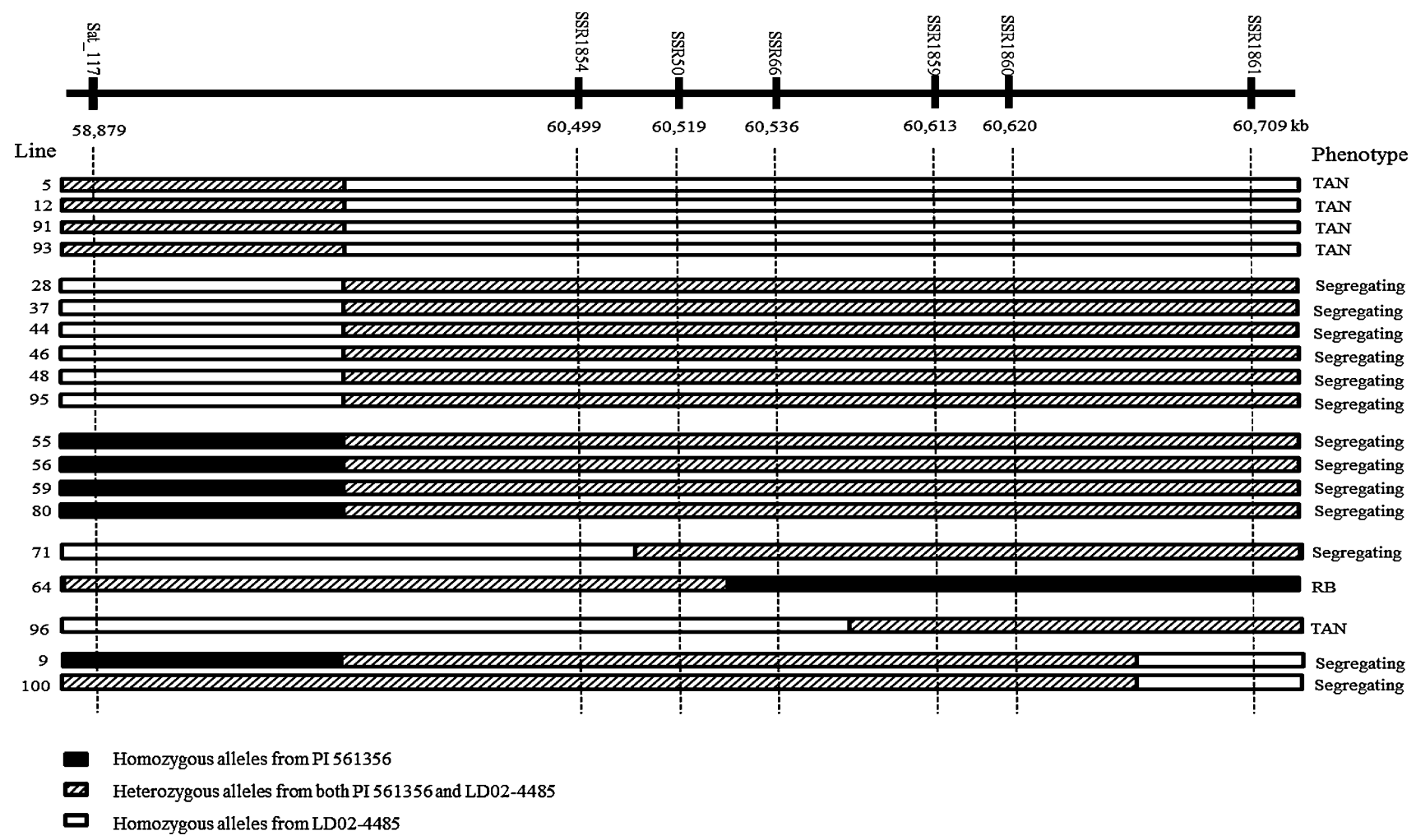

Fig. 1 Graphical genotypes of recombinant lines from PI $561356 \times$ LD02-4485 population in the soybean rust resistance gene interval on soybean chromosome 18. Phenotypes indicate the type of reaction to the $P$. pachyrhizi isolate ZM01-1. RB is the resistant and TAN the susceptible reaction. The lines with segregating phenotype

on chromosome 18. The SoySNP50 Infinium chip contains a total of 52,041 SNPs and the Infinium assay (Song et al. 2012) was used as per the manufacturer's instructions and analyzed using Illumina GenomeStudioV2010.2 software (Illumina, San Diego, CA). The SNP haplotype analysis was conducted using MEGA version 4 (Tamura et al. 2007). The distance between any pair of accessions was calculated based on the percentage of the SNPs carrying different alleles. If a SNP call was missing in one accession, the SNP was only deleted from the comparisons of the SNP in that one accession with its paired comparison with the other accessions and all other paired comparisons for that SNP were included. The neighbor-joining method was used for the construction of trees and a bootstrap with 1,000 replicates was used to measure confidence in the branch.

\section{Results}

The $P$. pachyrhizi isolate ZM01-1 produced TAN lesions on PI 200492 (Rppl), PI 462312 (Rpp3), G01-PR33 consisted of progeny with RB or TAN reaction. The physical positions of the markers $(\mathrm{kb})$ are based on soybean chromosome 18 sequence of the G. max genome (assembly version 1.01) available at http://soybase.org/gbrowse/cgi-bin/gbrowse/gmax 1.01/

[Rpp?(Hyuuga)] and Williams, while it did not overcome the resistance of PI 230970 (Rpp2) and PI 459025B (Rpp4) (Table 3). The responses of these soybean accessions were consistent with those previously observed by Chakraborty et al. (2009) after inoculation with the same isolate. The parents of the population, PI 561356 and LD02-4485, produced RB and TAN lesions to the ZM01-1 isolate, respectively. There were significant differences for disease severity among the SBR resistance sources. PI 561356 had significantly $(P=0.05)$ less disease severity than all of the resistance sources except PI 200492 (Rppl), and also had significantly less sporulation than all of the other resistance sources (Table 3). PI 230970 (Rpp2) and PI 459025B (Rpp4), which both gave RB reaction, had less sporulation than the other resistance sources with the TAN lesions (Table 3). These results show that SBR resistance in PI 561356 is more effective in controlling ZM01-1 than genotypes with Rpp2 and Rpp4.

The segregation of SBR lesion types (RB or TAN) for the $100 \mathrm{~F}_{2: 3}$ lines fit a 3 resistant: 1 susceptible segregation ratio $\left(\chi^{2}=0.12, P=0.73\right)$ when the homozygous resistant and segregating lines were combined into a single class 
Table 3 Reaction, disease severity, and sporulation of lines in the PI $561356 \times$ LD02-4485 population, and parents and checks after inoculation with the Phakopsora pachyrhizi isolate ZM01-1

\begin{tabular}{|c|c|c|c|}
\hline Genotype & Reaction & Disease severity $^{a}$ & Sporulation $^{\mathrm{b}}$ \\
\hline \multicolumn{4}{|l|}{ Checks and parents } \\
\hline PI 200492 (Rppl) & TAN & 2.6 & 5.0 \\
\hline L85-2378 (Rppl) & TAN & 3.3 & 5.0 \\
\hline PI 230970 (Rpp2) & $\mathrm{RB}$ & 3.3 & 2.9 \\
\hline PI 462312 (Rpp3) & TAN & 3.1 & 5.0 \\
\hline PI 459025B (Rpp4) & $\mathrm{RB}$ & 2.8 & 3.0 \\
\hline G01-PR33 [(Rpp?(Hyuuga)] & TAN & 2.9 & 5.0 \\
\hline Williams (susceptible) & TAN & 2.8 & 5.0 \\
\hline PI 561356(Rpp1-?) & $\mathrm{RB}$ & 2.4 & 1.2 \\
\hline LD02-4485 (susceptible) & TAN & 3.0 & 5.0 \\
\hline $\operatorname{LSD}(\alpha=0.05)$ & - & 0.4 & 0.3 \\
\hline \multicolumn{4}{|l|}{ Population } \\
\hline Population mean $(n=100)$ & $\mathrm{RB} / \mathrm{TAN}$ & 2.7 & 3.1 \\
\hline Homozygous RB line mean $(n=13)$ & $\mathrm{RB}$ & 2.2 & 1.1 \\
\hline Segregating line mean $(n=61)$ & $\mathrm{RB} / \mathrm{TAN}$ & 2.7 & 2.7 \\
\hline Homozygous TAN line mean $(n=26)$ & TAN & 3.1 & 5.0 \\
\hline
\end{tabular}

$R B$ reddish brown-colored lesions (resistant reaction), TAN pale brown-colored lesions (susceptible reaction)

${ }^{a}$ Disease severity on a scale of 1 (no visible lesions), 2 (light infection with few lesions present), 3 (light to moderate infection), 4 (moderate to severe infection), and 5 (prolific lesions)

${ }^{\mathrm{b}}$ Amount of uredinia sporulation within RB or TAN lesions. Sporulation on a scale of 1 (no sporulation), 2 (<25\% of the lesions sporulating), 3 (26-50\% of the lesions sporulating), 4 (51-75\% of the lesions sporulating), and 5 (76-100\% of the lesions sporulating). All TAN lesions were rated 5 for sporulation

(Table 4). However, when the homozygous resistant and segregating lines are separated, the segregation among the lines does not fit a 1:2:1 segregation $\left(\chi^{2}=8.22, P=0.02\right)$ (Table 4). The segregation of individual resistant and susceptible plants in the segregating lines also fit to 3 resistant: 1 susceptible ratio $\left(\chi^{2}=1.04, P=0.59\right)$, indicating that resistance from PI 561356 is controlled by a single, dominant gene.

Lines selected with $\mathrm{RB}$ and TAN lesions were used in BSA with markers linked to known SBR resistance genes. In the BSA, SSR markers near Rppl and Rpp4 were associated with the SBR lesion type. All lines in the population were then genotyped with all available polymorphic SSR markers near the two genes, and the SSR markers Satt503, Satt288, and Sat_117 near Rppl were genetically linked to lesion type while markers linked to Rpp4 were not associated with resistance. This shows that PI 561356 has a major SBR resistance gene in the Rppl region and that the PI does not have a major allele at Rpp4 or linked to Rpp4. Unfortunately, the SSR markers, Sct_187,Sat_064, and Sat_372, which were used in previous mapping of Rppl and Rppl-b, did not show polymorphisms between the two parents, PI 561356 and LD024485. To identify additional markers in the Rppl region that are polymorphic between our two parents, seven BARCSOYSSR markers located in this region (Song et al. 2010) as well as the several SSR markers designed based on super contig 112 of the Williams 82 sequence but not listed in Song et al. (2010) were tested. A total of six SSR markers, BARCSOYSSR_18_1854 (SSR1854), SSR50, SSR66, BARCSOYSSR_18_1859 (SSR1859), BARCSOYSSR_18_1860 (SSR1860) and BARCSOYSSR_18_1861 (SSR1861), were identified as polymorphic in this screening and all lines in the population were then genotyped with these SSR markers (Table 5).

All SSR markers used to map the resistance in the population fit a 3:1 segregation when those lines homozygous for the allele from the resistant parent and the segregating lines were combined in one class and compared to lines homozygous for the allele from the susceptible parent (Table 4). The segregation of Satt503, Satt288, and Sat_117 in the population fit a 1 resistant:2 segregating:1 susceptible ratio, but the SBR lesion type and the other markers did not due to the presence of fewer than expected homozygous resistant lines and more than expected heterozygous lines in the population (Table 4).

SBR lesion type in the population was mapped as a qualitative trait with the data from the population in which the homozygous resistant, segregating, and homozygous susceptible lines were in separate classes using JoinMap 3.0. This mapping placed the resistance gene from PI 561356 into a $1-\mathrm{cM}$ region on soybean chromosome 18 , flanked on one side by the marker SSR50 and on the other 
Table 4 Inheritance of soybean rust resistance (RB or TAN reaction) to the Phakopsora pachyrhizi isolate ZM01-1 and the

SSR markers used to map the

SBR resistance gene in the population of $100 \mathrm{~F}_{2: 3}$ lines from the cross PI $561356 \times$ LD02-4485

a 3 homozygous resistant $(\mathrm{R})$ and segregating $(\mathrm{H})$ lines: 1 homozygous susceptible (S) lines

b 1 Homozygous resistant (R) lines:2 segregating (H) lines:1 homozygous susceptible (S) lines

\begin{tabular}{lllll}
\hline Locus & Expected ratio & Observed ratio & Chi-square & Probability \\
\hline Satt503 & $3: 1^{\mathrm{a}}$ & $72: 28$ & 0.34 & 0.56 \\
Satt288 & $3: 1$ & $76: 24$ & 0.01 & 0.94 \\
Sat_117 & $3: 1$ & $72: 28$ & 0.34 & 0.56 \\
SSR1854 & $3: 1$ & $73: 27$ & 0.21 & 0.64 \\
SSR50 & $3: 1$ & $74: 26$ & 0.12 & 0.73 \\
SSR66 & $3: 1$ & $74: 26$ & 0.12 & 0.73 \\
SBR resistance & $3: 1$ & $74: 26$ & 0.12 & 0.73 \\
SSR1859 & $3: 1$ & $75: 25$ & 0.00 & 1.00 \\
SSR1860 & $3: 1$ & $75: 25$ & 0.00 & 1.00 \\
SSR1861 & $3: 1$ & $73: 27$ & 0.21 & 0.64 \\
Satt503 & $21: 51: 28$ & 1.00 & 0.61 \\
Satt288 & $1: 2: 1^{\mathrm{b}}$ & $21: 55: 24$ & 1.18 & 0.55 \\
Sat_117 & $1: 2: 1$ & $17: 55: 28$ & 3.42 & 0.18 \\
SSR1854 & $1: 2: 1$ & $12: 61: 27$ & 9.34 & 0.01 \\
SSR50 & $1: 2: 1$ & $12: 62: 26$ & 9.68 & 0.01 \\
SSR66 & $1: 2: 1$ & $13: 61: 26$ & 8.22 & 0.02 \\
SBR resistance & $1: 2: 1$ & $13: 61: 26$ & 8.22 & 0.02 \\
SSR1859 & $1: 2: 1$ & $13: 62: 25$ & 8.64 & 0.01 \\
SSR1860 & $1: 2: 1$ & $13: 62: 25$ & 8.64 & 0.01 \\
SSR1861 & $1: 2: 1$ & $13: 60: 27$ & 7.92 & 0.02 \\
\hline & $1: 2: 1$ & & &
\end{tabular}

Table 5 List of new SSR and BARCSOYSSR markers used to map the SBR resistance gene in PI 561356

\begin{tabular}{|c|c|c|c|c|c|}
\hline Name & Sequence ID & Product size $^{\mathrm{a}}$ & Primer & Sequences & Positions ${ }^{\mathrm{b}}$ \\
\hline SSR1854 & BARCSOYSSR_18_1854 & 183 & $\begin{array}{l}\text { Forward } \\
\text { Reverse }\end{array}$ & $\begin{array}{l}\text { TGGTTCTTTCAATTTAATATGC } \\
\text { AAGTAGAATGTACGAATTATTG }\end{array}$ & $60,499,775-60,499,957$ \\
\hline SSR50 & - & 226 & $\begin{array}{l}\text { Forward } \\
\text { Reverse }\end{array}$ & $\begin{array}{l}\text { AGCACTAACAACTTTCTTTG } \\
\text { GTTCTTAAATCTTACCСTCAC }\end{array}$ & $60,518,978-60,519,203$ \\
\hline SSR66 & - & 279 & $\begin{array}{l}\text { Forward } \\
\text { Reverse }\end{array}$ & $\begin{array}{l}\text { AGATTGGGTGAGAACATAAG } \\
\text { GGAGAGCGTAAAAGAAATTC }\end{array}$ & $60,535,339-60,535,617$ \\
\hline SSR1859 & BARCSOYSSR_18_1859 & 294 & $\begin{array}{l}\text { Forward } \\
\text { Reverse }\end{array}$ & $\begin{array}{l}\text { CTCAATCGCATCCTTGCATA } \\
\text { GCCTTCCAACTCATGTTTCAA }\end{array}$ & $60,613,084-60,613,377$ \\
\hline SSR 1860 & BARCSOYSSR_18_1860 & 145 & $\begin{array}{l}\text { Forward } \\
\text { Reverse }\end{array}$ & $\begin{array}{l}\text { AGACATTCGTTGCAAAAGCC } \\
\text { TTAGCCCTTCCCAAGAAACA }\end{array}$ & $60,619,935-60,620,079$ \\
\hline SSR1861 & BARCSOYSSR_18_1861 & 247 & $\begin{array}{l}\text { Forward } \\
\text { Reverse }\end{array}$ & $\begin{array}{l}\text { TGCCACAATGTCCACAACTT } \\
\text { CCCTTTTCTTTTGCCTCTCC }\end{array}$ & $60,709,612-60,709,858$ \\
\hline
\end{tabular}

a PCR product sizes are based on the G. max genome (assembly version 1.01) available http://soybase.org/gbrowse/cgi-bin/gbrowse/gmax1.01/

${ }^{\mathrm{b}}$ Physical position of the markers based on the G. max genome (assembly version 1.01) available at http://soybase.org/gbrowse/cgi-bin/gbrowse/ gmax 1.01/. The base pair (bp) positions of the markers correspond to the locations of forward and reverse primer on soybean chromosome 18

${ }^{c}$ BARCSOYSSR sequence ID are based on Song et al. (2010). Forward and reverse primer sequences of the SSR1854 in our study are different from the sequences in Song et al. (2010), although the SSR motifs are the same. The primer pairs used in the present study were first developed based on the sequence of super contig 112 before BARCSOYSSR_18_1854 was released by Song et al. (2010)

side by SSR1859 or SSR1860 (Fig. 1). No recombination was observed between SSR66 and SBR resistance as well as between SSR1859 and SSR1860. The lack of recombination between SSR1859 and SSR1860 is not surprising considering these two markers are only $6.7 \mathrm{~kb}$ apart based on the G. max genome (assembly version 1.01) (http://soybase.org). Graphical genotypes of the 19 lines with recombination events near the resistance gene also supported its location between SSR50 and SSR1859 (Fig. 1). The physical distance between SSR50 and SSR1859 based on the $G$. max genome (assembly version 1.01) is $94.4 \mathrm{~kb}$ (http://soybase.org).

There was a significant $\left(P<0.0001 ; R^{2}=0.70\right)$ association between SBR lesion type (RB or TAN) and disease severity in the population with homozygous RB lines having a severity rating of 2.2 and homozygous TAN lines 
having a rating of 3.1 (Table 3 ). Similarly, homozygous RB lines have significantly less sporulation than homozygous TAN lines (Table 3). Quantitative trait loci (QTL) controlling disease severity from PI 561356 were mapped using the interval mapping function in MapQTL 4.0 and the LOD peak for the trait mapped to the same interval as the SBR lesion type. SSR66 has the highest LOD score for disease severity (LOD $=26.2$ ) and the percentage of the variance explained by the segregation for the marker was 70.1. A QTL for sporulation was also mapped to the same interval (data not shown), which was expected because all lines in the population with a TAN reaction were given a sporulation rating of 5.0. This resulted in the sporulation and lesion type ratings being non-independent. The most likely explanation for these results is that the allele from PI 561356 that confers RB lesion type also confers reduced severity and sporulation, although we cannot completely exclude the possibility that these phenotypes are controlled by more than one gene.

Thirty-nine soybean accessions including the 33 major North American soybean ancestors, Williams 82, and 5 SBR resistance sources with resistance genes mapping to the Rppl region were analyzed to identify SNP haplotypes across a 213-kb interval on chromosome 18 that includes Rppl (Table 1). Twenty-one SNP markers located across this interval formed 21 distinct haplotypes including 4 haplotypes among the 5 SBR-resistant PIs (Table 1). PI 200492 (Rppl) belongs to SNP haplotype 16, PI 594538A (Rpplb) belongs to haplotype 1, PI 587886 and PI 587880A form haplotype 19, and PI 561356 belongs to haplotype 9. Haplotypes 16 and 19 were unique to these resistant sources, as none of the ancestral soybean ancestors had these haplotypes. In contrast, seven North American soybean ancestors belong to haplotype 1 along with PI 594538A (Rppl-b) and the four ancestors belong to haplotype 9 with PI 561356 (Table 1). Haplotype analysis using only the eight SNP markers located within the 94.4-kb region between SSR50 and SSR1859 where the SBR resistance gene from PI 561356 mapped formed 16 haplotypes (Fig. 2). Although there were changes in the total number of haplotypes compared to the result from the $213-\mathrm{kb}$ region analysis, the five SBR resistance sources formed the same four unique haplotypes. One key difference was that PI 548461 (Improved Pelican) was grouped to the same haplotype with PI 561356 in the 94.4-kb region analysis (Fig. 2). There are a total of nine SNPs (haplotype tags) that are unique for specific haplotypes among the five SBR resistance sources in the 213$\mathrm{kb}$ region and five of them are located in the 94.4-kb region (Table 1). A dendrogram based on the eight SNP markers in the 94.4-kb region surrounding Rppl showed clustering of the resistance sources PI 587886 and PI 587880A, which was expected because they had the same haplotype. The other sources did not cluster suggesting that these resis- tance alleles are not the result of a recent divergence from an ancestral Rppl allele.

The three SSR markers (SSR50, SSR66, and SSR1859) that flank or co-segregate with the SBR resistance gene in PI 561356 could distinguish the SBR resistance sources and SSR66 and SSR1859 could distinguish the SBR resistance sources from the ancestral accessions better than the SNP markers (Table 1; Fig. 3). The SSR marker SSR50 separated the five SBR resistance sources into three groups, and SSR66 and SSR1859 separated these resistance sources into two groups (Table 1; Fig. 3). SSR1859 was the only marker that separated the resistance sources into groups that included none of the ancestral sources. A pattern similar to that observed for the SNP haplotypes was found with three markers for PI 200492. This was the only accession with resistance to FL07-1 and it had a unique allele for the markers compared to the other resistance sources (Table 2; Fig. 3). SSR66 was also predictive of resistance to ZM01-1, as this marker gave unique alleles to PI 594538A, PI 587886, PI 587880A and PI 561356, which are the only accessions with resistance to this $P$. pachyrhizi isolate (Table 2; Fig. 3). The PIC values of SSR50, SSR66, and SSR 1859 were $0.83,0.85$, and 0.50 , respectively.

The 5 SBR resistance sources, Williams 82, and 11 North American soybean ancestors belonging to SNP haplotype 1 or 9 , which were chosen because resistance sources had these haplotypes, were evaluated with the $P$. pachyrhizi isolates, FL07-1 and ZM01-1, to test for an association between SBR resistance and SNP haplotypes or SSR marker genotypes (Table 2). The only evidence of an association between SNP haplotypes and resistant reactions was that PI 200492 (Rppl, haplotype 16) had a unique haplotype and was the only accession that showed a resistant reaction to FL07-1 (Table 2). There was no association between haplotypes and reactions to ZM01-1, as the SBRresistant sources PI 594538A, PI 587886, PI 587880A, and PI 561356 showed RB reactions, while all other accessions, including accessions with the same haplotype as PI 594538A and PI 561356, gave TAN reactions (Table 2).

\section{Discussion}

The SBR resistance gene controlling lesion type (RB or TAN), disease severity, and sporulation from PI 561356 was mapped onto soybean chromosome 18 in this study. SBR resistance alleles from the other four soybean accessions were previously mapped to the same region on chromosome 18 (Hyten et al. 2007; Chakraborty et al. 2009; Ray et al. 2009). Although we could not use the same markers as in the previous studies, the physical locations of these markers on the G. max genome (assembly version 1.01) (http://soybase.org) could be directly compared. 
Fig. 2 Dendrogram showing similarity coefficients and genetic relationships among 39 soybean genotypes analyzed by eight SNPs in a 94.4-kb region between SSR50 and SSR1859 on chromosome 18 . The digits at nodes are the percent occurrence in 1,000 bootstrap replications

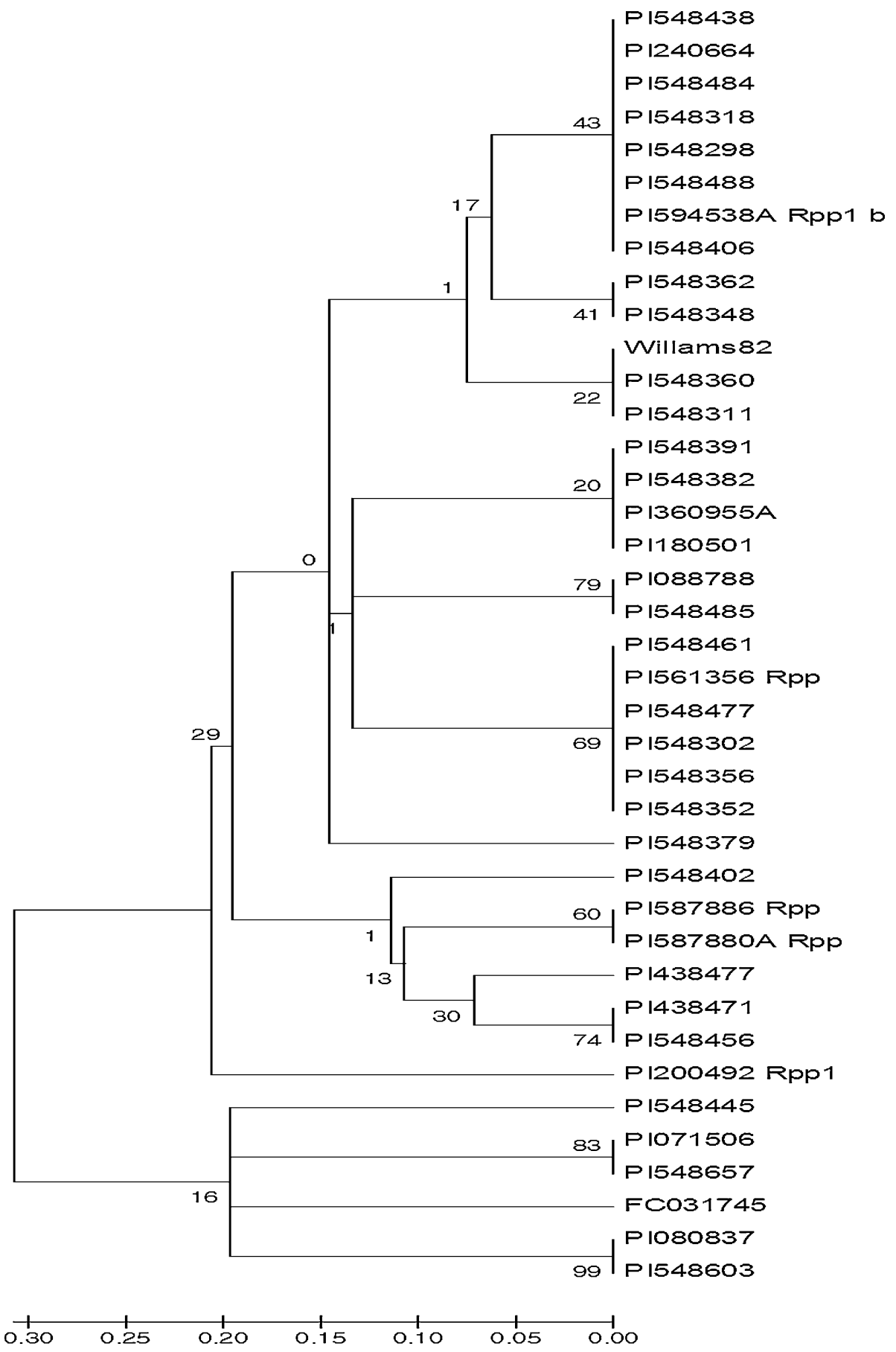

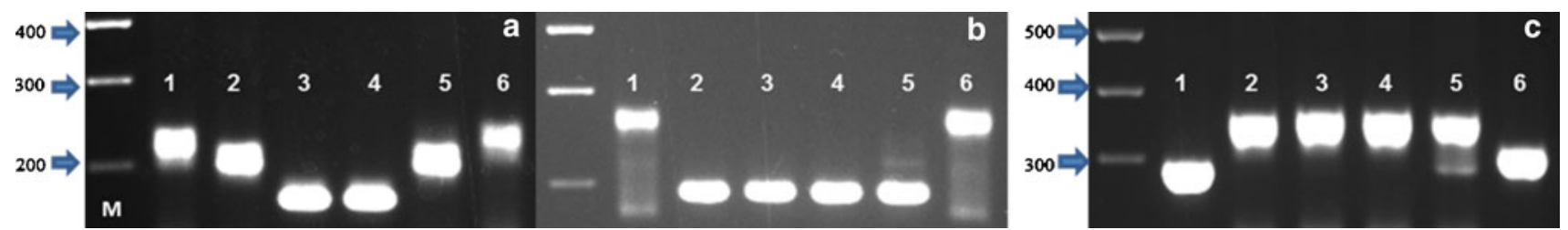

Fig. 3 Agarose gel electrophoresis of SSR50 (a), SSR66 (b), and SSR1859 (c) for the five soybean rust-resistant soybean accessions and Williams 82. $M$ molecular size marker. Size markers are the 200, 300,
400, and $500 \mathrm{bp}$ fragments of a 1-kb DNA ladder. Lane 1 PI 200492 (Rppl), lane 2 PI 594538A (Rpp1-b), lane 3 PI 587886, lane 4 PI 587880A, lane 5 PI 561356, and lane 6 Williams82 


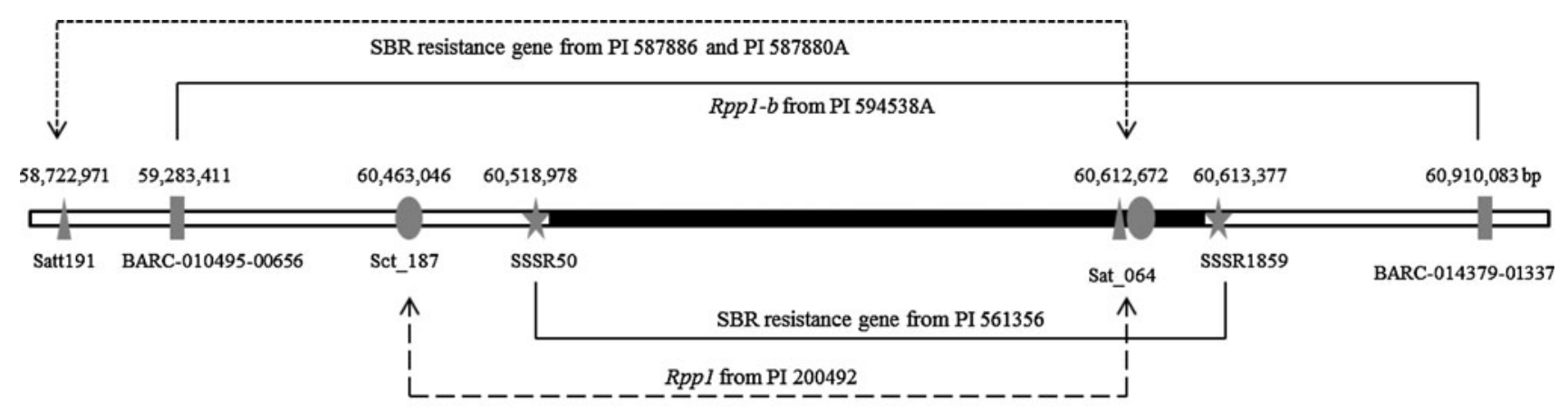

SSR markers used to map SBR resistance gene from PI 587886 and 587880A

SNP markers used to map Rppl-b from PI 594538A

SSR markers used to map Rppl from PI 200492

SSR markers used to map SBR resistance gene from PI 561356

Fig. 4 Physical positions of the soybean rust resistance gene regions from five soybean accessions with the genes that map to the Rppl region on chromosome 18. Physical positions of the flanking SSR or SNP markers used to genetically map Rppl from PI 200492 (Hyten et al. 2007), Rppl-b from PI 594538A (Chakraborty et al. 2009), the

Hyten et al. (2007) reported that Rppl from PI 200492 was mapped to a $149.6-\mathrm{kb}$ interval between Sct_187 and Sat_064, Chakraborty et al. (2009) reported that Rpp1$b$ from PI 594538A mapped to a $1,626.7-\mathrm{kb}$ interval between BARC-010495-00656 and BARC-01437901337, and Ray et al. (2009) mapped alleles from two PIs to a $1,889.7-\mathrm{kb}$ interval between Satt191 and Sat_064 (Fig. 4). We mapped the SBR resistance gene from PI 561356 to a $94.4-\mathrm{kb}$ interval between SSR50 $(60,518,978)$ and SSR1859 (60,613,311) (Figs. 1; 4). A comparison of the interval that resistance genes were mapped across the five sources reveals an overlapping 93.6-kb interval between SSR50 and Sat_064 that resistance from each source maps. Therefore, it is possible that all five sources have a resistance allele at the same locus in this interval (Fig. 4). It is still possible, however, that resistance alleles from these sources could be at different tightly linked loci. Fine mapping or cloning of the resistance genes from these sources will be required to separate these two possibilities.

Schmutz et al. (2010) reported that the average ratio of genetic-to-physical distance is $1 \mathrm{cM}$ per $197 \mathrm{~kb}$ in euchromatic regions and $1 \mathrm{cM}$ per $3.5 \mathrm{Mb}$ in heterochromatic regions in soybean. Ninety-three percent of the recombination occurs in repeat-poor, gene-rich euchromatic genomic regions that only accounts for $43 \%$ of the genome. The Rppl region is located in a gene-rich interval outside the pericentromeric region on chromosome 18 (http://soybase.org/gbrowse/cgi-bin/gbrowse/gmax1.01). In our study, the SBR resistance gene from PI 561356 was genetically mapped to a 1-cM interval which corresponds to a
SBR resistance gene from PI 587886 and PI 587880A (Ray et al. 2009), and the SBR resistance gene from PI 561356 are based on soybean chromosome 18 sequence of the G. $\max$ genome (assembly version 1.01) available at http://soybase.org/gbrowse/cgi-bin/gbrowse/ $\operatorname{gmax} 1$

94.4-kb physical distance on Williams 82 chromosome 18 (http://soybase.org). The ratio of genetic-to-physical distances of the Rppl region in PI 200492 was $187 \mathrm{~kb} \mathrm{cM}^{-1}$, $R p p l-b$ in PI 594538A was $290 \mathrm{~kb} \mathrm{cM}^{-1}$, and the SBR resistance gene regions in PI 587886 and PI $857880 \mathrm{~A}$ was $116 \mathrm{~kb} \mathrm{cM}^{-1}$. Differences in the genetic-to-physical distances in these regions are likely the result of the small genetic distances sampled and relatively large errors in these genetic distance estimates caused by the small populations used in these studies. The relatively high recombination rate in the Rppl interval suggests that this would be a good candidate region for positional cloning.

The SBR resistance gene from PI 594538A was given the allelic designation $R p p 1-b$ because, although it mapped to the Rpp1 region, the gene from PI 594538A gave an RB response to the $P$. pachyrhizi isolate ZM01-1, while Rpp1 produced a TAN reaction (Chakraborty et al. 2009). The resistance reactions of PI 561356 were the same as PI 594538A for ZM01-1, FL07-1, and the four isolate mixture tested by Miles et al. (2006). This consistency of resistance reactions and mapping of resistance genes to the same interval suggests that these two sources may have the same resistance allele. The SBR resistance genes in the Rppl region from PI 587886 and PI 587880A were found to have a similar resistance pattern as PI 594538A for FL07-1 and ZM01-1 in our test, and for six additional $P$. pachyrhizi isolates in a test reported by Ray et al. (2009). There is some evidence suggesting that PI 587886 and PI 587880A may not have Rppl-b, as one isolate tested by Ray et al. (2009) gave a mixed IM/RB reaction for PI 587886 and PI 587880A, while PI 594538A gave a RB reaction. Miles 
et al. (2006) showed that both PI 561356 and PI 594538A gave mixed reactions to a mixture of four $P$. pachyrhizi isolates, while PI 587886 and PI 587880A gave RB reactions.

The relative genetic positions of the markers used to map the SBR resistance gene in PI 561356 were consistent with their genetic and physical locations on the G. max consensus map 4.0 and the G. $\max$ genome (assembly version 1.01) (http://soybase.org; Figs. 1; 4). The current gene annotation of the 94.4-kb region between SSR50 and SSR1859 on the G. max genome (assembly version 1.01) predicts the presence of nine high-confidence genes (verified 16 August 2011). Of the predicted genes, Glyma18g51700 and Glyma18g51750 are nucleotide-binding site (NBS) - leucine-rich repeat (LRR) genes encoding a leucine-rich transmembrane protein. Nucleotide and peptide similarity between the two genes are 93 and $88 \%$, respectively (http://soybase.org; http://www.phytozome. net). Their protein sequences share homology with BED finger NBS-LRR resistance protein in black cottonwood (Populus trichocarpa Torr. \& Gray) (E value: $2 \mathrm{e}-118$ ) and putative disease resistance protein RPM1 in castor bean (Ricinus communis L.) (E value: $4 \mathrm{e}-100$ ) (http:// www.ncbi.nlm.nih.gov/). There also is evidence that SBR resistance genes in soybean might be in the NBS-LRR gene family, as Meyer et al. (2009) identified an NBS-LRR as a single candidate gene for Rpp4 in PI 459025B. Monteros et al. (2010) narrowed the Rpp?(Hyuuga) interval down to a 371-kb region and reported that this region also included NBS-LRR and LRR genes. Although Glyma18g51700 and Glyma18g51750 are good candidate genes for SBR resistance in PI 561356, the gene annotation is from Williams 82 , which does not have resistance to SBR. Therefore, this gene and other candidate genes in the region need to be cloned from their resistance sources and complemented in a susceptible background to identify the coding sequence for Rppl.

SNP haplotype analyses were previously conducted across intervals containing Rpp?(Hyuuga) and Rpp3, which both map to the same region on chromosome 6 . Monteros et al. (2010) tested a 800-kb region where Rpp?(Hyuuga) maps with five SNP markers and Hyten et al. (2009) tested a 897-kb interval which includes Rpp3 from PI 462312 with 275 SNPs. In both studies, there was no haplotype tag that could differentiate Rpp?(Hyuuga) from Rpp3, which was previously mapped to the same region on chromosome 6 by Hyten et al. (2009). In our study, 21 SNP markers mapping within the Rpp1 region produced 4 distinct SNP haplotypes among the 5 Rppl sources, as well as nine haplotype tags, were identified (Table 1).

There was no clear association between the SNP haplotypes in the Rppl region, the origin of the accessions, or SBR resistance reactions to two $P$. pachyrhizi isolates (Tables 1, 2). PI 200492 originated from Shikoku, Japan, while PI 594538A was collected from Fujian, China, and PI 587886, PI 587880A, and PI 561356 were from Zhejiang, China. Haplotype 1 included PI 594538A and seven North American soybean ancestors and these were collected from China, North Korea, or the Philippines. Four North American ancestral genotypes and PI 561356 belonged to haplotype 9 and these were collected from China, Japan, or North Korea (Table 1). Although these 11 major North American soybean ancestors belong to the same SNP haplotypes with PI 594538A or PI 561356, they produced TAN lesions to both P. pachyrhizi isolates, while PI 594538A and PI 561356 produced RB lesions to ZM01-1 (Table 2).

There were no SNP markers or haplotypes that could distinguish between the 5 SBR-resistant accessions and the 33 susceptible ancestral accessions. In contrast, the SSR markers were more informative than the SNP markers and SSR 1859 produced PCR products unique for only SBR resistance sources (Table 1; Fig. 3). SSR1859 produced two differently sized PCR products among the five resistance sources tested and these were different from those produced by any of the susceptible accessions. The marker SSR66 could differentiate the resistance sources, except PI 200492 (Rpp1), from the 33 major North American soybean ancestors (Table 1). The allele from PI 200492 is shared with Williams 82 and many other susceptible accessions. There were no SNP and SSR markers in the Rppl region that could differentiate between PI 587886 and PI 587880A, suggesting that these two PIs may have the same resistance allele in the Rppl region (Table 1; Fig. 3). These results suggest that SSR66 and SSR1859 could be useful in predicting whether SBRresistant accessions with unknown resistance genes have the same resistance allele in the Rppl region as the five known sources used in the current study.

PI 200492 has a unique SNP haplotype, SSR genotype, and resistance response compared to the other four resistance sources with resistance in the Rppl region. This indicates that the Rppl region from PI 200492 has undergone a separate evolutionary path compared to the other sources with resistance genes mapping to the Rppl region.

Although single SBR resistance genes such as Rpp 1, 1$b, 2,3,4,5$, and 6 can be introduced into North American elite cultivars by backcrossing in a relatively short time, $P$. pachyrhizi isolates have overcome or will likely overcome any single gene resistance in the future. Therefore, it is important to continue screenings to identify novel SBR resistance sources. Breeding the right combination of resistance alleles at Rppl and other loci including alleles for partial resistance using the flanking markers may lead to increased durability of resistance to SBR and could be effectively used in the other soybean breeding programs. 
Acknowledgments This research was supported by funding from the United Soybean Board.

\section{References}

Born H, Diver S (2005) Asian soybean rust: notes and organic control options for farmers. http://www.agrisk.umn.edu/cache/ARL0 2950.htm

Botstein D, White RL, Skolnick M, Davis RW (1980) Construction of genetic linkage map in man using restriction fragment length polymorphisms. Am J Hum Genet 32:314-331

Bromfield KR (1984) Soybean rust. Monograph no. 11. American Phytopathological Society, St. Paul

Caldwell P, McLaren NW (2004) Soybean rust research in South Africa. In: Proc. VII world soybean res. conf. IV int. soybean processing and utilization conference, III Brazilian soybean congress, Foz do Iguassu, PR, Brazil, pp 354-360

Chakraborty N, Curley J, Frederick RD, Hyten DL, Nelson RL, Hartman GL, Diers BW (2009) Mapping and confirmation of a new allele at Rpp 1 from soybean PI 594538A conferring RB lesion-type resistance to soybean rust. Crop Sci 49:783-790

Fan JB, Chee MS, Gunderson KL (2006) Highly parallel genomic assays. Nat Rev Genet 7:632-644

Fehr WR, Caviness CE, Burmood DT, Pennington JS (1971) Stage of development descriptions for soybeans, Glycine max (L.) Merrill. Crop Sci 11:929-931

Garcia A, Calvo ES, Kiihl RS, Harada A, Hiromoto DM, Vieira LGE (2008) Molecular mapping of soybean rust (Phakopsora pachyrhizi) resistance genes: discovery of a novel locus and alleles. Theor Appl Genet 117:545-553

Gizlice Z, Carter TE, Burton JW (1994) Genetic base for North American public soybean cultivars released between 1947 and 1988. Crop Sci 34:1143-1151

Gore MA, Chia JM, Elshire RJ, Sun Q, Ersoz ES, Hurwitz BL, Peiffer JA, McMullen MD, Grills GS, Ross-Ibarra J, Ware DH, Buckler ES (2009) A first-generation haplotype map of maize. Science 326:1115-1117

Hartman GL, Wang TC, Tschanz AT (1991) Soybean rust development and the quantitative relationship between rust severity and soybean yield. Plant Dis 75:596-600

Hartman GL, Miles MR, Frederick RD (2005) Breeding for resistance to soybean rust. Plant Dis 89:664-666

Hartman GL, Hill CB, Twizeyimana M, Miles MR, Bandyopadhyay R (2011) Interaction of soybean and Phakopsora pachyrhizi, the cause of soybean rust. CAB Rev Perspect Agric Vet Sci Nutr Nat Resour 6. doi:10.1079/PAVSNNR20116025

Hennings VP (1903) A few new Japanese Uredinaceae. Hedwigia 42:S107-S108

Hershman D, Sikora E, Giesler L (2011) Soybean rust PIPE: Past, present, and future. J Integr Pest Manag 2. doi:10.1603/IPM11001

Hyten DL, Song Q, Zhu Y, Choi IY, Nelson RL, Costa JM, Specht JE, Shoemaker RC, Cregan PB (2006) Impacts of genetic bottlenecks on soybean genome diversity. Proc Natl Acad Sci USA 103:16666-16671

Hyten DL, Hartman GL, Nelson RL, Frederick RD, Concibido VC, Narvel JM, Cregan PB (2007) Map location of the Rpp 1 locus that confers resistance to soybean rust in soybean. Crop Sci 47:837-840

Hyten DL, Smith JR, Frederick RD, Tuker ML, Song Q, Cregan PB (2009) Bulk segregate analysis using the GoldenGate assay to locate the Rpp 3 locus that confers resistance to Phakopsora pachyrhizi (soybean rust) in soybean. Crop Sci 49:265-271
Isard SA, Gage SH, Comtois P, Russo JM (2005) Principles of the atmospheric pathway for invasive species applied to soybean rust. Bioscience 55:851-862

Johnson GC, Esposito L, Barratt BJ, Smith AN, Heward J, Genova GD, Ueda H, Cordell HJ, Eaves IA, Dudbridge F, Twells RC, Payne F, Hughes W, Nutland S, Stevens H, Carr P, TuomilehtoWolf E, Tuomilehto J, Gough SC, Clayton DG, Todd JA (2001) Haplotype tagging for the identification of common disease genes. Nat Genet 29:233-237

Keim P, Olson T, Shoemaker RC (1988) A rapid protocol for isolating soybean DNA. Soybean Genet News1 15:150-152

Killgore E, Heu R (1994) First report of soybean rust in Hawaii. Plant Dis 78:1216

Lam HM, Xu X, Liu X, Chen W, Yang G, Wong FL, Li MW, He W, Qin N, Wang B, Li J, Jian M, Wang J, Shao G, Wang J, Sun SS, Zhang G (2010) Resequencing of 31 wild and cultivated soybean genomes identifies patterns of genetic diversity and selection. Nat Genet 42:1053-1059

Li S, Smith JR, Ray JD, Frederick RD (2012) Identification of a new soybean rust resistance genes in PI 567102B. Theor Appl Genet 125:133-142

Melching JS, Bromfield KR, Kingsolver CH (1983) The plant pathogen containment facility at Frederick, Maryland. Plant Dis 67:717-722

Meyer JDF, Silva DCG, Yang C, Pedley KF, Zhang C, van de Mortel M, Hill JH, Shoemaker RC, Abdelnoor RV, Whitham SA, Graham MA (2009) Identification and analyses of candidate genes for Rpp 4-mediated resistance to Asian soybean rust in soybean (Glycine $\max$ (L.) Merr.). Plant Physiol 150:295-307

Michelmore RW, Paran I, Kesseli RV (1991) Identification of markers linked to disease resistance genes by bulked segregant analysis: a rapid method to detect markers in specific genomic regions by using segregating populations. Proc Natl Acad Sci USA 88:98289832

Miles MR, Frederick RD, Hartman GL (2003) Soybean rust: is the U.S. soybean crop at risk? APS Net Plant Pathol. doi:10.1094/APSnetFeature-2003-060

Miles MR, Frederick RD, Hartman GL (2006) Evaluation of soybean germplasm for resistance to Phakopsora pachyrhizi. Plant Health Prog. doi:10.1094/PHP-2006-0104-01-RS

Miles MR, Bonde MR, Nester SE, Berner DK, Frederick RD, Hartman GL (2011) Characterizing resistance to Phakopsora pachyrhizi in soybean. Plant Dis 95:577-581

Monteros MJ, Missaoui AM, Phillips DV, Walker DR, Boerma HR (2007) Mapping and confirmation of the 'Hyuuga' red-brown lesion resistance gene for Asian soybean rust. Crop Sci 47:829-836

Monteros MJ, Ha BK, Phillips DV, Boerma HR (2010) SNP assay to detect the 'Hyuuga' red-brown lesion resistance gene for Asian soybean rust. Theor Appl Genet 121:1023-1032

Mueller TA, Miles MR, Morel W, Marios JJ, Wright DL, Kemerait RC, Levy C, Hartman GL (2009) Effect of fungicide and timing of application on soybean rust severity and yield. Plant Dis 93:243-248

Pathan MS, Sleper DA (2008) Advances in soybean breeding. In: Stacey G (ed) Genetics and genomics of soybean. Springer, NY

Pham TA, Miles MR, Frederick RD, Hill CB, Hartman GL (2009) Differential responses of resistant soybean entries to isolates of Phakopsora pachyrhizi. Plant Dis 93:224-228

Rafalski A (2002) Applications of single nucleotide polymorphisms in crop genetics. Curr Opin Plant Biol 5:94-100

Ray JD, Morel W, Smith JR, Frederick RD, Miles MR (2009) Genetics and mapping of adult plant rust resistance in soybean PI 587886 and PI 587880A. Theor Appl Genet 119:271-280 
Ribeiro AS, Ubirajara J, Moreira V, Pierozzi EB, Rachid BF, Toledo JFF, Arias CAA, Soares RM, Godoy CV (2007) Genetic control of Asian rust in soybean. Euphytica 157:15-25

SAS Institute (2002) The SAS system for Windows. Release 9.2. SAS Institute, Cary

Schmutz J, Cannon S, Schlueter J, Ma J, Mitros T, Nelson W, Hyten D, Song Q, Thelen J, Cheng J, Xu D, Hellsten U, May G, Yu Y, Sakurai T, Umezawa T, Bhattacharyya M, Sandhu D, Valliyodan B, Lindquist E, Peto M, Grant D, Shu S, Goodstein D, Barry K, Futrell-Griggs M, Du J, Tian Z, Zhu L, Gill N, Joshi T, Libault M, Sethuraman A, Zhang X, Shinozaki K, Nguyen H, Wing R, Cregan P, Specht J, Grimwood J, Rokhsar D, Stacey G, Shoemaker R, Jackson S (2010) Genome sequence of the paleopolyploid soybean. Nature 463:178-183

Schneider RW, Hollier CA, Whitman HK, Palm ME, McKenny JM, Hernández JR, Levy L, Devries-Paterson R (2005) First report of soybean rust caused by Phakopsora pachyrhizi in the continental United States. Plant Dis 89:774

Shastry BS (2004) Role of SNP/haplotype map in gene discovery and drug development: an overview. Drug Dev Res 62:143-150

Silva DCG, Yamanaka N, Brogin RL, Arias CAA, Nepomuceno AL, Di Mauro AO, Pereira SS, Nogueira LM, Passianotto ALL, Abdelnoor RV (2008) Molecular mapping of two loci that confer resistance to Asian rust in soybean. Theor Appl Genet 117:57-63

Song QJ, Jia GF, Zhu YL, Grant D, Nelson RT, Hwang EY, Hyten DL, Cregan PB (2010) Abundance of SSR motifs and development of candidate polymorphic SSR markers (BARCSOYSSR_1.0) in soybean. Crop Sci 50:1950-1960

Song QJ, Hyten DL, Jia GF, Quigley C, Fickus E, Cregan PB (2012) Development and Evaluation of a high-density Illumina Infinium iSelect Beadchip SoySNP50K. In: Plant and animal genome conference XX. San Diego, CA, USA. http://pag.confex.com/pag/xx/ webprogram/Paper4683.html
Tamura K, Dudley J, Ne Mi, Kumar S (2007) MEGA4: molecular evolutionary genetics analysis (MEGA) software version 4.0. Mol Biol Evol 24(8):1596-1599

USDA-ARS (2012) National Genetic Resources Program. Germplasm Resources Information Network-(GRIN). (Online Database) National Germplasm Resources Laboratory, Beltsville, Maryland. Retrieved from http://www.ars-grin.gov/npgs/index.html

Utomo HS, Linscombe SD (2009) Current patents and future development underlying marker-assisted breeding in major grain crops. Rec Pat DNA Gene Seq 3:53-62

Van Ooijen JW, Voorrips RW (2001) Joinmap 3.0. Software for the calculation of genetic linkage maps. Plant Research International, Wageningen

Van Ooijen JW, Boer MP, Jansen RC, Maliepaard C (2002) MapQTL 4.0. Software for the calculation of QTL positions on genetic maps. Plant Research International, Wageningen

Wang D, Shi J, Carlson SR, Cregan PB, Ward RW, Diers BW (2003) A low-cost, high-throughput polyacrylamide gel electrophoresis system for genotyping with microsatellite DNA markers. Crop Sci 43:1828-1832

Yorinori JT, Paiva WM, Frederick RD, Costamilan LM, Bertagnoli PF, Hartman GL, Godoy CV, Nunes JJ (2005) Epidemics of soybean rust (Phakopsora pachyrhizi) in Brazil and Paraguay from 2001 to 2003. Plant Dis 89:675-677

Zhu YL, Song QJ, Hyten DL, Van Tassell CP, Matukumalli LK, Grimm DR, Hyatt SM, Fickus EW, Young ND, Cregan PB (2003) Single-nucleotide polymorphisms in soybean. Genetics 163:1123-1134

Zhu Q, Zheng X, Luo J, Gaut BS, Ge S (2007) Multilocus analysis of nucleotide variation of Oryza sativa and its wild relatives: severe bottleneck during domestication of rice. Mol Biol Evol 24:875888 